\title{
Parametric resonances in electrostatically interacting carbon nanotube arrays
}

\author{
A. Isacsson* and J. M. Kinaret \\ Department of Applied Physics, Chalmers University of Technology, SE-412 96 Göteborg, Sweden \\ (Received 19 December 2008; revised manuscript received 26 February 2009; published 14 April 2009)
}

\begin{abstract}
We study, numerically and analytically, a model of a one-dimensional array of carbon nanotube resonators in a two-terminal configuration. The system is brought into resonance upon application of an ac signal superimposed on a dc bias voltage. When the tubes in the array are close to each other, electrostatic interactions between tubes become important for the array dynamics. We show that both transverse and longitudinal parametric resonances can be excited in addition to primary resonances. The intertube electrostatic interactions couple modes in orthogonal directions and affect the mode stability.
\end{abstract}

DOI: 10.1103/PhysRevB.79.165418

PACS number(s): 85.85. $+\mathrm{j}, 85.35 . \mathrm{Kt}$

\section{INTRODUCTION}

During recent years, several experimental realizations of nanoelectromechanical (NEM) resonators based on carbon nanotubes (CNTs) or carbon nanofibers (CNFs) have been demonstrated. ${ }^{1-6}$ Carbon nanotubes have established themselves as strong material candidates for use in NEMresonator systems, partly due to their favorable mechanical properties $^{7}$ such as low mass and high elastic modulus. Thus, using CNTs or CNFs allows for operational frequencies of NEM resonator that reach into the gigahertz regime. In the most recent experiments, the long predicted high-quality factors on the order of $Q \sim 10^{3}$ have finally been achieved. This makes these resonators interesting from a technological point of view with application areas such as tunable rf filters and fast low-power switching elements. ${ }^{8-10}$ However, for such applications, a major drawback is the high impedance levels offered by single-nanotube devices. The ensuing low-power transduction ${ }^{8}$ makes integration of such devices with current state-of-the-art complementary metal-oxide semiconductor (CMOS) technology difficult. One way to overcome this problem is to construct devices based on parallel arrays. For arrays it is desirable to know how interactions between elements affect the operation of devices. It is, for instance, important to know how closely spaced array members can be placed without drastic changes in performance.

Apart from the technological incentive of studying microelectromechanical (MEM) and NEM arrays, the problem is also of fundamental interest. The dynamic response of coupled NEM- and MEM-resonator systems in combination with nonlinearities is known to lead to unexpected and/or unintuitive behavior. Examples are intrinsic localized modes ${ }^{11-14}$ and mode synchronization. ${ }^{15,16}$ In addition, parametric resonances in MEM and NEM arrays have been studied both experimentally and theoretically. ${ }^{17-21}$ In those studies parametric response was induced by applying an acvoltage component between alternating beams in beam arrays. Parametric resonances can be narrower than fundamental resonances and find use in, for instance, parametric amplifiers.

In this paper we study theoretically a vertical onedimensional (1D) regular array of CNT or CNF resonators (see Fig. 1). This configuration is natural if one wishes to make, for instance, a frequency-tunable rf filter and wishes to overcome impedance mismatching problems. ${ }^{6,23}$ As in Ref. 6 this layout is suitable for capacitive actuation and transduction. When the resonators are not too widely separated, electrostatic interactions between the tubes become important and affect the dynamical response of the system. While the system considered in this paper shares some features with previously studied systems, ${ }^{17-21}$ there are several important differences. Among them two are worthy of special attention. First, all the tubes in the system are connected to the same voltage source, containing both a dc and an ac component, thereby eliminating the need for individual contacting of alternating array members. This makes the interactions between tubes repulsive, rather than attractive. Second, in contrast to beams with rectangular cross sections, where the characteristic vibration frequencies differ for vibrations in different directions, CNTs or CNFs have a circular cross section and motion in two dimensions plays an important role.

We find that the fundamental resonance, where the tubes oscillate in unison toward the drain electrode, is not drastically affected by interactions. However, several other resonances appear including longitudinal resonances, where the tubes oscillate in the direction along the array. For small

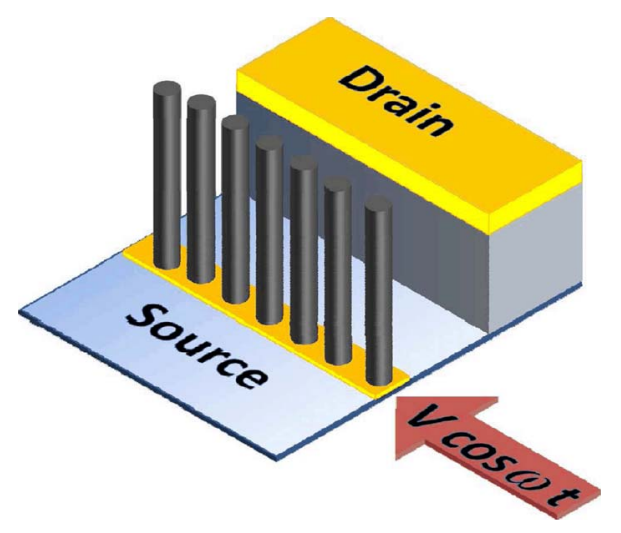

FIG. 1. (Color online) Schematic layout of a vertically oriented carbon nanotube NEM array. Through patterning of catalysts, regular one-dimensional arrays of carbon nanotubes or nanofibers can be grown on top of an electrode (source). A second electrode (drain) is formed from a deposited layer at the same height as the tube tips. Actuation and transduction of tube displacement are achieved through electrostatic (capacitive) coupling between the tubes and the drain electrode. 
arrays, these resonances have the form of hardening Duffingtype resonances which develop into a band of resonances as the array gets larger. Further, two parametric resonances are present in the systems. Both transverse and longitudinal motions may be parametrically excited, with both multiple branches and complex bifurcation structures for the larger arrays. The electrostatic coupling between tubes also affects the stability of the longitudinal motion, which becomes unstable due to parametric excitation of transverse oscillations.

We begin this paper by presenting, in Sec. II, a simplified lumped electromechanical model to derive the main qualitative features of the system. Then, in Sec. III, based on numerical integration of the equations of motion, the general features of the dynamic response of a one-dimensional CNT resonator array are discussed. To better understand the main characteristics of this response, we focus on the smallest possible array (two tubes) in Sec. IV. The two-tube system is treated both numerically and analytically. We derive frequency response equations and analyze stability for the various resonances using perturbation theory. These analytical results are found to work as good approximations for finding the loci of the resonances also in larger systems. This treatment is then followed up, in Sec. V, with a discussion of how the response changes as the arrays become larger before concluding in Sec. VI.

\section{MODEL}

For an array consisting of $N$ tubes we denote the coordinates of the central axis of each (undeformed) tube by $\mathbf{X}_{i}^{0}$ $=\left(X_{i}, Y_{i}\right)$, where $i=1, \ldots, N$. For the vibrations of the beams we consider only excitations of the fundamental flexural modes for which a lumped model is suitable. ${ }^{9,22}$ Describing the position of the tip of the cantilevers by the coordinates $\mathbf{X}_{i}$, we use the following equation of motion: ${ }^{23}$

$$
m_{i} \ddot{\mathbf{X}}_{i}+m_{i} \gamma \dot{\mathbf{X}}_{i}+m_{i} \omega_{0 i}^{2}\left(\mathbf{X}_{i}-\mathbf{X}_{i}^{0}\right)=\mathbf{F}_{i}^{\mathrm{el}}, \quad i=1, \ldots, N .
$$

Here $m_{i}$ are the effective masses of the tubes and $\omega_{0 i}$ are the natural resonance frequencies. For tubes with circular cross sections these values are given by ${ }^{23} m_{i}=\rho A_{i} L_{i} / 5.684$ and $\omega_{0 i}=3.516 L_{i}^{-2} \sqrt{E I_{i} / \rho_{i} A_{i}}$, where $A_{i}$ is the cross-sectional area, $I_{i}$ is the moment of inertia, $E$ is Young's modulus, and $L_{i}$ is the length of tube number $i$. We have also introduced a viscoelastic damping term $\gamma_{i}$ for each tube to account for mechanical losses. In the absence of a gaseous medium surrounding the tubes, the main sources of dissipation are external, such as clamping losses and Ohmic losses, and we will use a typical $Q$ of 100 . We note here that numerical studies $^{24}$ show that the intrinsic losses in carbon nanotube cantilever resonators are at least 1 order of magnitude smaller.

Aside from elastic forces, external electrostatic forces $\mathbf{F}_{i}^{\mathrm{el}}$ act on the tubes. These forces depend on the geometry as well as the instantaneous charge distributions on the tubes. To find the exact charge distributions on the tubes is a hard problem. Numerical simulations using the finite element method and boundary element method of electrostatically interacting tubes ${ }^{25}$ reveal that the charge is mainly located at the tip of the tubes. Furthermore, the main contributions to the bending moments arise from forces close to the tube tips. Thus to model the electrostatic forces we make the simplified assumption that the charge $Q_{i}$ on each tube is concentrated to a conducting spherical shell located at the tip of each tube. The model we consider is one of conducting spheres, attached with springs to their equilibrium positions and able to move in the $x y$ plane. The drain electrode, which can be taken to be at zero potential, is modeled as an infinite conducting plane. There is also the possibility of actually having metallic grains on top of the tubes. In plasma chemical-vapor deposition (CVD) growth of CNT or CNF from Ni catalysts, tip growth results in the metallic catalysts residing at the tips of the tubes.

In general, the full charge distribution, rather than the total charge, on each sphere is needed to find the forces. Provided that the intertube separation and the tube-drain separations are larger than the tube diameters, the dominant contribution to the electrostatic forces comes from the monopole contributions of these charge distributions. Restricting attention to this case the electrostatic free energy of the system [the tubes being biased by the common time-dependent voltage $V(t)]$ is

$$
\mathcal{F}_{\text {el }}=\mathcal{F}_{\text {self }}+\mathcal{F}_{\text {int }}-V(t) \sum_{i=1}^{N} Q_{i},
$$

where the electrostatic self-energy is

$$
\mathcal{F}_{\text {self }}=\frac{1}{4 \pi \epsilon_{0}} \sum_{i=1}^{N} \frac{Q_{i}^{2}}{D_{i}}
$$

and the total electrostatic interaction energy is

$$
\mathcal{F}_{\text {int }}=\frac{1}{4 \pi \epsilon_{0}} \sum_{i=1}^{N} \sum_{j>i}^{N} \frac{Q_{i} Q_{j}}{\left|\mathbf{X}_{i}-\mathbf{X}_{j}\right|}-\frac{1}{8 \pi \epsilon_{0}} \sum_{i, j=1}^{N} \frac{Q_{i} Q_{j}}{\left|\mathbf{X}_{i}^{\prime}-\mathbf{X}_{j}\right|} .
$$

Here $\mathbf{X}_{i}^{\prime}=\left(-X_{i}, Y_{i}\right)$ denote the images of $\mathbf{X}_{i}=\left(X_{i}, Y_{i}\right)$ in the plane $X=0$. The charge distribution in the array is then found from solving the linear system $\partial \mathcal{F}_{\mathrm{el}} / \partial Q_{i}=0$, after which the electrostatic forces may be found as $\mathbf{F}_{i}=-\nabla_{\mathbf{x}_{i}} \mathcal{F}_{\text {el }}$.

We consider now a uniform system with identical tubes, i.e., $D_{i}=D, \omega_{0 i}=\omega_{0}, \gamma_{i}=\gamma$, and $m_{i}=m$. Rescaling the coordinates to dimensionless form according to $X_{i}=D x_{i}, Y_{i}=D y_{i}$ yields

$$
\ddot{\mathbf{x}}_{i}+\gamma \dot{\mathbf{x}}_{i}+\omega_{0}^{2}\left(\mathbf{x}_{i}-\mathbf{x}_{i}^{0}\right)=\frac{1}{m D} \mathbf{F}_{i}^{\mathrm{el}} .
$$

In the same way we rescale electric quantities through introducing a unit voltage $V_{0}$, i.e., $V=v V_{0}, Q_{i}=q_{i} 4 \pi \epsilon_{0} D V_{0}$, and the electrostatic charging energy $\mathcal{E}_{C}=4 \pi \epsilon_{0} D V_{0}^{2}$. The corresponding dimensionless electrostatic free energy $f_{\mathrm{el}}$ $\equiv \mathcal{F}_{\text {el }} / \mathcal{E}_{C}$ is then

$$
f_{\mathrm{el}}=\sum_{i=1}^{N} q_{i}\left(q_{i}-v\right)+\sum_{i=1}^{N} \sum_{j>i}^{N} \frac{q_{i} q_{j}}{\left|\mathbf{x}_{i}-\mathbf{x}_{j}\right|}-\frac{1}{2} \sum_{i, j=1}^{N} \frac{q_{i} q_{j}}{\left|\mathbf{x}_{i}^{\prime}-\mathbf{x}_{j}\right|} .
$$

In order to study the response of the system we solve dynamic equation (1) numerically using a velocity Verlet algorithm. In Sec. III we present the main qualitative features 

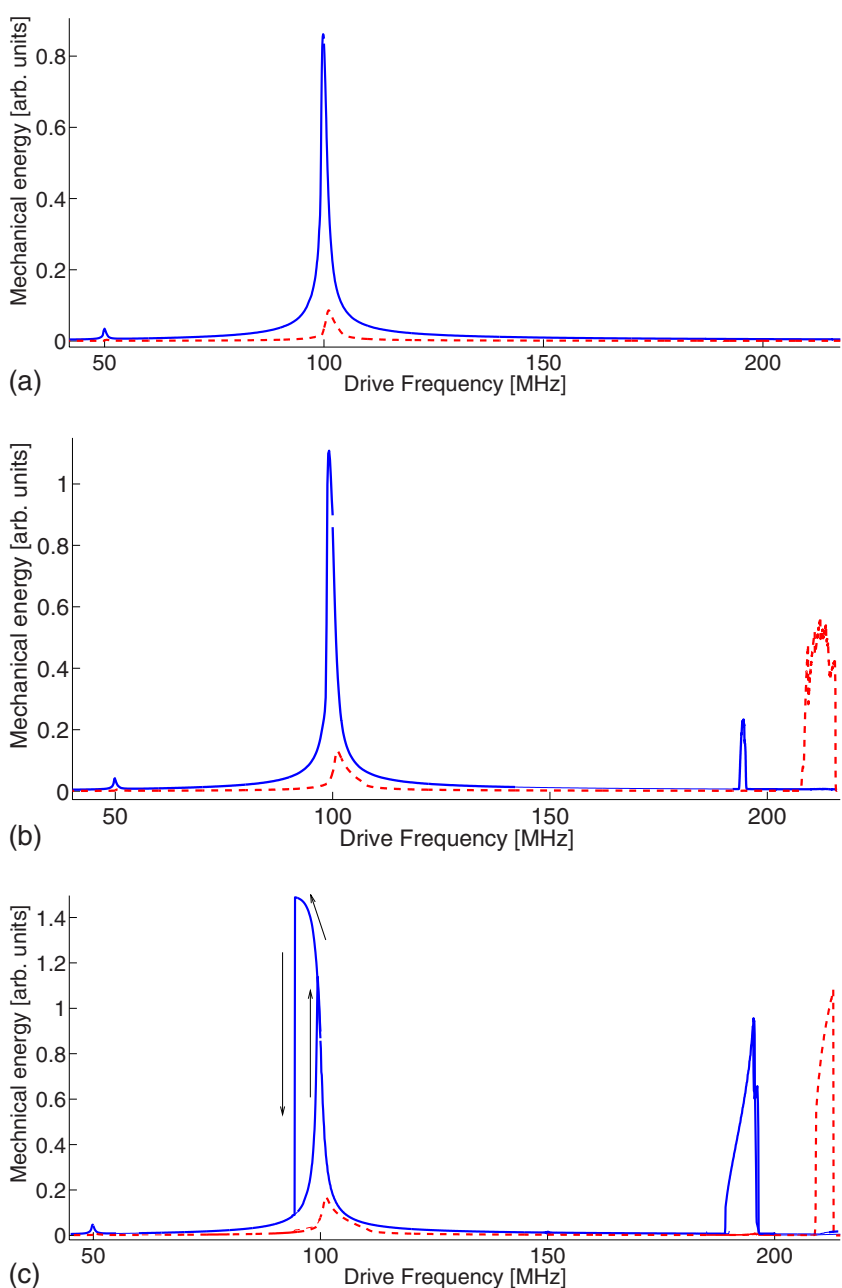

FIG. 2. (Color online) Time-averaged mechanical energy for a harmonically driven array with 100 tubes. The blue solid lines represent the mechanical energy stored in transverse modes (vibrations toward the drain electrode). The red dashed lines represent the energy stored in longitudinal modes (vibration along the array axis). Arrows indicate the directions of transitions in the hysteretic region. Top: intertube separation of $200 \mathrm{~nm}$. Middle: intertube separation of $150 \mathrm{~nm}$. Bottom: intertube separation of $125 \mathrm{~nm}$.

of the mechanical response of the system to a harmonic driving field. A more quantitative discussion is then carried out in Secs. IV and V.

\section{RESPONSE TO HARMONIC DRIVING}

Figure 2 shows the response of an array with 100 tubes when it is driven with an ac signal on the source in combination with a static dc bias voltage $V(t)=V_{0}+V_{1} \cos \left(\omega_{D} t\right)(\mathrm{dc}$ bias voltage $V_{0}=12 \mathrm{~V}$, ac signal $V_{1}=2 \mathrm{~V}$ ). The tubes are each $1 \mu \mathrm{m}$ long with a diameter of $25 \mathrm{~nm}$. The distance to the drain electrode is $150 \mathrm{~nm}$. Plotted are the two orthogonal components of the mechanical energy corresponding to transverse (blue solid line) and longitudinal vibrations (red dashed lines). The energy is scaled in terms of the dimensionless units introduced above with a time scale set to $t_{0}=0.1 \mathrm{~ns}$.
In the top panel the spacing between the tubes is $200 \mathrm{~nm}$. Clearly visible is the primary transverse resonance (blue lines) where all the tubes oscillate in phase with each other. This mode corresponds to the resonance of a single nanotube. A band of longitudinal modes can be seen (dashed line) just above $100 \mathrm{MHz}$. In the middle panel the tubes are more closely spaced $(150 \mathrm{~nm})$ and two additional resonances are present. The transverse resonance (around $190 \mathrm{MHz}$ ) is a parametrically excited resonance where each tube oscillates with half the driving frequency. In this resonance neighboring tubes oscillate with opposing phases (optical mode). Above $210 \mathrm{MHz}$ is another parametric resonance in the more closely spaced arrays. This is a parametric resonance of the band of longitudinal modes of the array. In the bottom panel the spacing has been narrowed down further to $125 \mathrm{~nm}$. The parametric resonances are now stronger and the primary transverse resonance has become hysteretic. The appearance of hysteresis can here be understood by considering the attractive force between a tube and the drain electrode. For widely separated tubes, each tube is attracted by its own image potential alone, while for more closely spaced tubes, the images charges from neighboring tubes contribute to this force.

In Sec. IV we show how these resonances and their main characteristics can be understood from analyzing a twooscillator array. Then, in Sec. V we study, numerically, how the response changes qualitatively as the size of the arrays grow larger. The parametric resonances, both the transverse and the longitudinal, show a complex behavior with multiple bifurcation points. These are not shown in the panels of Fig. 2 but will be addressed further in Sec. V.

\section{TWO OSCILLATORS: CASE STUDY}

In this section we study the simplest case, namely, an array consisting of only two cantilevers with identical physical parameters. This case can be analyzed analytically and serves to validate numerical modeling and provides insights for larger arrays.

We take the positions of the undeflected tubes to be $\mathbf{x}_{1}^{0}$ $=\left(x_{0}, y_{0}\right)$ and $\mathbf{x}_{2}^{0}=\left(x_{0},-y_{0}\right)$, where $x_{0}<0$ and $y_{0}>0$, and the drain to be the plane $x=0$. Introducing the variables $x_{ \pm}$ $=x_{1} \pm x_{2}$ and $y=y_{1}-y_{2}$, we have the equations of motion

$$
\begin{gathered}
\ddot{x}_{+}+\gamma \dot{x}_{+}+\omega_{0}^{2}\left(x_{+}-2 x_{0}\right)=\nu \omega_{0}^{2} v^{2}\left[\frac{g_{1}^{2}}{\left(x_{+}+x_{-}\right)^{2}}+\frac{g_{2}^{2}}{\left(x_{+}-x_{-}\right)^{2}}\right. \\
\left.\quad-2 g_{1} g_{2} \frac{x_{+}}{\left(x_{+}^{2}+y^{2}\right)^{3 / 2}}\right], \\
\ddot{x}+_{-}+\gamma \dot{x}_{-}+\omega_{0}^{2} x_{-}= \\
\nu \omega_{0}^{2} v^{2}\left[\frac{g_{1}^{2}}{\left(x_{+}+x_{-}\right)^{2}}-\frac{g_{2}^{2}}{\left(x_{+}-x_{-}\right)^{2}}\right. \\
\left.+2 g_{1} g_{2} \frac{x_{-}}{\left(x_{-}^{2}+y^{2}\right)^{3 / 2}}\right],
\end{gathered}
$$




$$
\begin{aligned}
\ddot{y}+\gamma \dot{y}+\omega_{0}^{2}\left(y+2 y_{0}\right)= & 2 \nu \omega_{0}^{2} v^{2} g_{1} g_{2}\left[\frac{y}{\left(x_{-}^{2}+y^{2}\right)^{3 / 2}}\right. \\
& \left.-\frac{y}{\left(x_{+}^{2}+y^{2}\right)^{3 / 2}}\right] .
\end{aligned}
$$

The relevant electromechanical coupling constant is $\nu$ $=\mathcal{E}_{C} / \mathcal{E}_{\text {mech }}=\mathcal{E}_{C} / m D^{2} \omega_{0}^{2}$. In terms of numbers $\nu$ $\approx 10^{-3} V_{0}^{2} L^{3} D^{-5}$ if length is measured in $\mathrm{nm}$ and the tubes are assumed solid with a Young's modulus of $1 \mathrm{TPa}^{26}$ The functions $g_{1,2}$ are found by solving exactly the electrostatic problem and are given by

$$
\begin{aligned}
& g_{1}=\left(x_{+}+x_{-}\right) \frac{\left(x_{+}-x_{-}\right)(\Delta-2)-1}{\left(x_{+}^{2}-x_{-}^{2}\right)\left(\Delta^{2}-4\right)-4 x_{+}-1}, \\
& g_{2}=\left(x_{+}-x_{-}\right) \frac{\left(x_{+}+x_{-}\right)(\Delta-2)-1}{\left(x_{+}^{2}-x_{-}^{2}\right)\left(\Delta^{2}-4\right)-4 x_{+}-1},
\end{aligned}
$$

where $\Delta$ is defined as

$$
\Delta=\frac{1}{\sqrt{x_{-}^{2}+y^{2}}}-\frac{1}{\sqrt{x_{+}^{2}+y^{2}}}=\Delta_{-}-\Delta_{+} .
$$

A spectrum that reveals the most important features of the response to a harmonic ac drive on the gate is shown in Fig. 3. This figure was obtained from numerical integration of the dynamic equations for a system with the following parameters: tube diameter $D=25 \mathrm{~nm}$; tube lengths $L=1 \mu \mathrm{m}$; bare quality factor $\kappa_{0}=100$; tube positions $\left(X_{0}, Y_{0}\right)=($ $-150,62.5) \mathrm{nm}$; Young's modulus $E=1 \mathrm{TPa}$; and tube density $\rho=1.2 \mathrm{~g} / \mathrm{cm}^{3}$. The applied voltages to the system were $V_{0}=12 \mathrm{~V}$ and $V_{1}=2 \mathrm{~V}$.

The figure was obtained by sweeping the drive frequency both upward and downward. On the vertical axis of Fig. 3 the dimensionless average mechanical energy of the tubes is shown. The motions in the longitudinal direction ( $y$ direction) and the transverse direction ( $x$ direction) have been separated for clarity. Both the transverse response (blue line) and the longitudinal response (red) show three main peaks each. We have labeled these peaks $\mathrm{A}, \mathrm{A}^{\prime}$, and $\mathrm{C}$ and $\mathrm{B}, \mathrm{B}^{\prime}$, and $\mathrm{D}$, respectively. Hysteresis in the frequency plane is present in peaks $\mathrm{B}, \mathrm{C}$, and $\mathrm{D}$ (for peak $\mathrm{C}$ the hysteresis is too narrow to be clearly seen in Fig. 3). In Secs. IV A-IV D below we treat each of these resonances in more detail. Note that in these subsections labels follow the labeling of the peaks in Fig. 3.

We begin by determining the stationary points. For small deflections around equilibrium it is sufficient to keep only the dominant terms in $1 / x_{+}$and $1 / y$ which yield the dynamic equations

$$
\begin{aligned}
& \ddot{x}_{+}+\gamma \dot{x}_{+}+\omega_{0}^{2}\left(x_{+}-2 x_{0}\right)=\nu \frac{\omega_{0}^{2} v^{2}}{2}\left[\frac{1}{x_{+}^{2}}-\frac{x_{+}}{\left(x_{+}^{2}+y^{2}\right)^{3 / 2}}\right], \\
& \ddot{y}+\gamma \dot{y}+\omega_{0}^{2}\left(y+2 y_{0}\right)=\nu \frac{\omega_{0}^{2} v^{2}}{2}\left[-\frac{1}{y^{2}}-\frac{y}{\left(x_{+}^{2}+y^{2}\right)^{3 / 2}}\right] .
\end{aligned}
$$

In the limit of large intertube separation $(y \rightarrow \infty)$ one retains the result of noninteracting tubes, whereas the limit $x \rightarrow \infty$

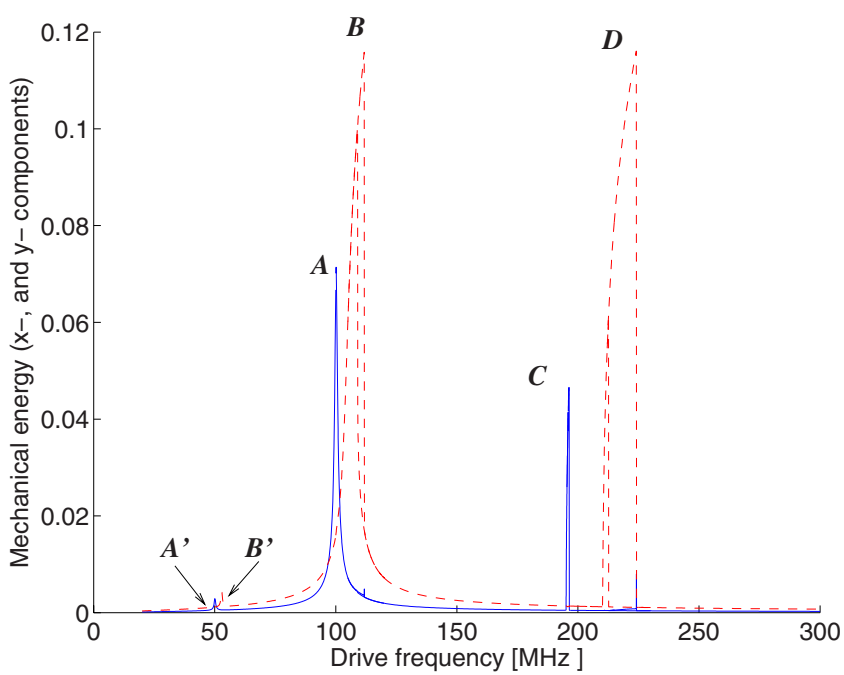

FIG. 3. (Color online) Average mechanical energy of a two-tube system in response to harmonic driving. Blue solid lines: average mechanical energy stored in vibrations in the $x$ direction. Red dashed lines: average mechanical energy stored in vibrations in the $y$ direction. Both upward and downward sweeps in frequency were made. $\mathrm{A}$ and $\mathrm{A}^{\prime}$ : fundamental resonances in the $x$ direction. Both tubes oscillate in phase toward the drain electrode ( $x_{+}$resonance). $\mathrm{B}$ and $\mathrm{B}^{\prime}$ : fundamental resonances in the $y$ direction. The tubes oscillate with opposing phases in the direction parallel to the drain electrode ( $y$ resonance). C: parametric resonance in the $x$ direction. The tubes oscillate with opposite phases at half the driving frequency in the direction toward drain electrode ( $x_{-}$resonance). D: parametric resonances in the $y$ direction. The tubes oscillate with opposing phases at half the driving frequency in the direction parallel to the drain electrode ( $y$ resonance).

reduces the problem to one in the $y$ direction only. The equations also decouple in the limit of small $\left|x_{+}\right|$(recall that both $x_{+}$and $y$ are negative) due to screening of the electrostatic interaction between the tubes by the drain electrode. System (5) can be used to determine the stationary deflections $x_{+}(t)$ $=x_{s}$ and $y(t)=y_{s}$ in the absence of an ac component. These time-independent solutions are found by solving the system

$$
\begin{gathered}
x_{s}=2 x_{0}+\frac{\nu}{2}\left[\frac{1}{x_{s}^{2}}-\frac{x_{s}}{\left(x_{s}^{2}+y_{s}^{2}\right)^{3 / 2}}\right], \\
y_{s}=-2 y_{0}+\frac{\nu}{2}\left[-\frac{1}{y_{s}^{2}}-\frac{y_{s}}{\left(x_{s}^{2}+y_{s}^{2}\right)^{3 / 2}}\right] .
\end{gathered}
$$

For small biases $v_{0}$ (i.e., $\left.\nu \ll 1\right)$ we solve perturbatively in $\nu$ and get

$$
\begin{aligned}
& x_{s} \approx 2 x_{0}\left[1-\frac{\nu}{16 y_{0}^{3}}\left(\sigma_{0}^{-3}+\Sigma_{0}\right)\right], \\
& y_{s} \approx-2 y_{0}\left[1+\frac{\nu}{16 y_{0}^{3}}\left(1-\Sigma_{0}\right)\right],
\end{aligned}
$$

where $\sigma_{0}=\left|x_{0} / y_{0}\right|$ and $\Sigma_{0} \equiv\left(1+\sigma_{0}^{2}\right)^{-3 / 2}$. The term $\Sigma_{0}$ accounts for mutual screening of the tubes (if $\sigma_{0}=\left|x_{0} / y_{0}\right| \rightarrow 0$ then $\left.\Sigma_{0} \rightarrow 1\right)$. This approximation is valid for small static 


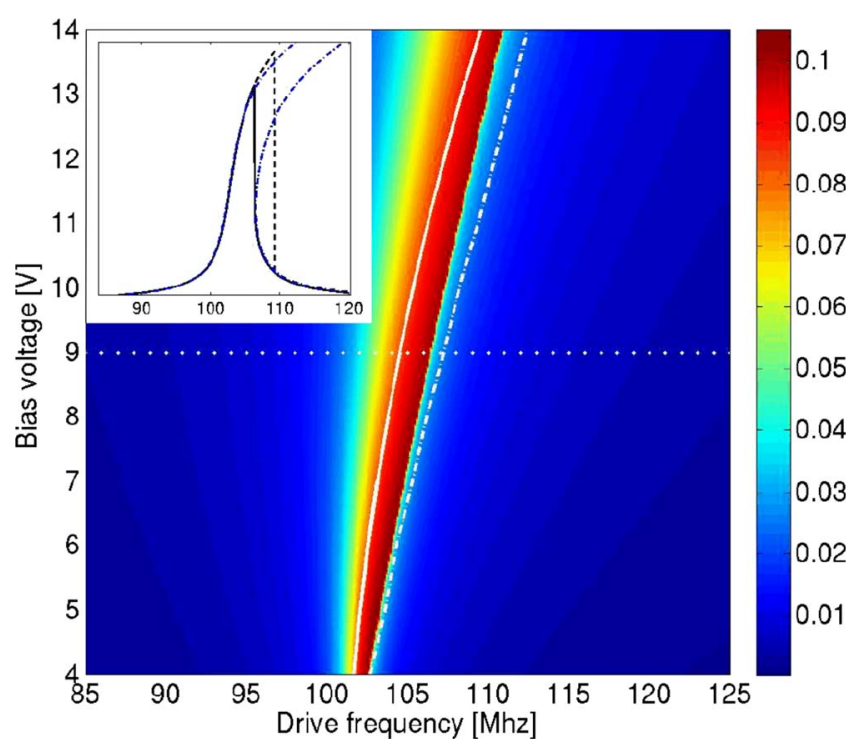

FIG. 4. (Color online) False color plot of mechanical energy of the primary longitudinal resonance ( $y$ resonance) as a function of bias voltage (vertical axis) and drive frequency (horizontal axis). The figure was created by sweeping the frequency downward. The solid white line corresponds to expression (12) for the central frequency $\omega_{c}^{(y)}$. Sweeping the frequency downward results in an abrupt change in the response at the bifurcation frequency $\omega_{B}^{(y)}$ [the highfrequency edge of the large amplitude (red) region]. The dashed white line shows the bifurcation point found from solving frequency response equation (13). The inset shows the response along the cross section at a bias of $9 \mathrm{~V}$ (along the dotted line). The thick black curves come from numerical simulations, whereas the dashdotted blue lines are solutions to frequency response equation (13).

deflections and far away from snap-in (the tubes making contact with the drain). Note that system (7) rests on the approximations $\left|1 / x_{+}\right| \ll 1$ and $|1 / y| \ll 1$. This is consistent with the approximation of only keeping the monopole contribution to the total charge distribution on the tube tips.

\section{A. Fundamental transverse resonance $\left(x_{+}\right.$resonance)}

In the fundamental transverse resonances ( $\mathrm{A}$ and $\mathrm{A}^{\prime}$ in Fig. 3) the tubes oscillate in phase with each other. Neither of these resonances differs appreciably in nature from those of single-tube systems. The subharmonic arises from the double frequency component of the driving occurring due to the $v^{2}$ term. The main resonance tunes downward in frequency with increasing bias and has a Duffing-type nonlinearity of the softening kind. We omit the analysis of this resonance in this paper since it has been already thoroughly studied previously in the literature in conjunction with single-cantilever resonators (see, for instance, Ref. 23).

\section{B. Fundamental longitudinal resonance (y resonance)}

The fundamental $y$ resonance (resonance B in Fig. 3) has the shape of a Duffing resonance with a hardening nonlinearity. A clearer view of the resonance is seen in the inset of Fig. 4. In this figure a false color plot of the mechanical energy in the primary longitudinal resonance ( $y$ resonance) as a function of bias voltage (vertical axis) and drive frequency (horizontal axis) is shown. The figure was created sweeping the frequency downward. Clearly visible is the upward tuning of resonance frequency with increasing bias and the sharp onset of resonance at the bifurcation point. The inset shows the response along the $9 \mathrm{~V}$ bias cross section (along the dotted line). The thick black curves are the results from numerical simulations of dynamic equation (1). The solid black line corresponds to downward frequency sweep, while the dashed line corresponds to upward frequency sweep.

The characteristics of the fundamental $y$ resonance can be found using perturbation theory. Considering this resonance we take $x_{+}=x_{s}$ and assume $x_{-}=0$. When $x_{-}=0$ the product $g_{1} g_{2}$ simplifies to

$$
g_{1} g_{2}=\frac{1}{\left[\Delta+2+x_{+}^{-1}\right]^{2}} .
$$

For small oscillations we may further set

$$
1 / \sqrt{x_{s}^{2}+y^{2}} \approx 1 / \sqrt{x_{s}^{2}+y_{s}^{2}}=\alpha .
$$

To obtain an estimate of the parametric dependence of the resonance character, we analyze the system using the method of averaging ${ }^{28}$ by making the ansatz $y(t)=y_{s}+Y(t) \cos \left(\omega_{D} t\right)$ in response to a drive given by $v(t)^{2} \approx v_{0}^{2}\left[1+2 \epsilon \cos \left(\omega_{D} t\right.\right.$ $+\delta)]$. Assuming $|\dot{Y} / Y| \ll \omega_{0}$, the differential equation for the amplitude $Y$ is

$$
\begin{gathered}
y_{s}=-2 y_{0}-\frac{\nu}{2} K_{0}\left(Y, y_{s}\right), \\
\dot{Y}=\omega_{0} \nu \frac{\epsilon}{2} \sin \delta\left[K_{2}\left(Y, y_{s}\right)-K_{0}\left(Y, y_{s}\right)\right]-\frac{1}{2} \gamma Y, \\
Y \omega_{D}=Y \omega_{0}+\frac{1}{2} \omega_{0} \nu\left(K_{1}+\epsilon \cos \delta\left[K_{0}+K_{2}\right]\right) .
\end{gathered}
$$

Here

$$
K_{n}\left(Y, y_{s}\right) \equiv \frac{2}{\pi} \int_{0}^{2 \pi} d \phi \cos n \phi \frac{A^{2}\left(1+\alpha^{3} y^{3}\right)}{\left(y_{s}+Y \cos \phi-A\right)^{2}},
$$

with $A \equiv\left(2+x_{s}^{-1}-\alpha\right)^{-1}$.

For small oscillation amplitudes where the response does not bifurcate, we solve the system to first order in $\nu$ in the limit $Y \rightarrow 0$. For the amplitude $Y$ and the center of resonance $\omega_{c}^{(y)}$, we get

$$
\begin{gathered}
Y=\frac{\omega_{0} \nu \epsilon\left(1-\Sigma_{0}\right)}{8 y_{0}^{2} \sqrt{\left(\omega_{D}-\omega_{c}^{(y)}\right)^{2}+\frac{\gamma^{2}}{4}},} \\
\frac{\omega_{c}^{(y)}}{\omega_{0}}=1+\frac{\nu}{16 y_{0}^{3}}\left[1+\left(\frac{1}{2} \sigma_{0}^{2}-1\right) \Sigma_{0}^{5 / 3}\right] .
\end{gathered}
$$

Equation (12) is useful for estimating the center frequency even for large oscillations as can be seen in Fig. 4, where $\omega_{c}^{(y)}$ obtained from Eq. (12) is drawn as the solid white line. 
As the drive gets stronger bifurcation occurs (see Fig. 3) and the shape of the resonance is found from solving the frequency response equation

$$
\omega_{0}^{2} \nu^{2} \epsilon^{2}=\frac{\gamma^{2} Y^{2}}{\left[K_{2}-K_{0}\right]^{2}}+\frac{\left[2 Y\left(\omega_{D}-\omega_{0}\right)-\omega_{0} \nu K_{1}\right]^{2}}{\left[K_{0}+K_{2}\right]^{2}} .
$$

The blue dash-dotted lines in Fig. 4 depict the resonances obtained from Eq. (13), showing good agreement for small amplitudes. The locus of the bifurcation point $\omega_{B}^{(y)}$ can also be determined from solving Eq. (13), and this solution for $\omega_{B}^{(y)}$ is shown as the white dashed line in Fig. 4.

Whereas both the location of the resonance and the bifurcation point can be estimated using Eq. (10), this is not true for finding the extent of the hysteresis. The destabilization of the high-amplitude branch is connected with an instability toward resonance of the $x_{-}$mode. The process of destabilization is depicted in Fig. 5, where the entire time evolutions of the trajectories of the tubes are shown. Starting in panel (A), the system is in the high-amplitude branch. The instability toward resonance of the $x_{-}$mode causes an increase in motion in the transverse direction [panels (B) and (C)]. Decay to the lower branch [panel (F)] occurs through irregular motion of the tubes [panels (D) and (E)]. The location where the high-amplitude branch of the $y$ resonance becomes unstable can be found analytically using perturbation theory. In Fig. 6 the region of instability toward parametric excitation of the $x_{-}$resonance is shown as the red dash-dotted line. As can be seen, a good estimate of the locus of the destabilization can be determined. The perturbative analysis is found in the Appendix.

\section{Instability toward parametric resonance $\left(x_{-}\right.$resonance)}

We now turn our attention to the parametric resonance of the $x_{-}$mode (resonance $\mathrm{C}$ in Fig. 3). Writing out explicitly the right-hand side of equation of motion (3), we have

$$
\ddot{x}_{-}+\gamma \dot{x}_{-}+\omega_{0}^{2} x_{-}=-2 x_{-} \omega_{0}^{2}\left[1+2 \epsilon \cos \left(\omega_{D} t\right)\right] F\left(x_{-}\right),
$$

with

$$
F=\nu\left(\frac{2 x_{s}(\Delta-2)^{2}-2(\Delta-2)}{\left[\left(x_{s}^{2}-x_{-}^{2}\right)\left(\Delta^{2}-4\right)-4 x_{s}-1\right]^{2}}-\frac{g_{1} g_{2}}{\left(x_{-}^{2}+y_{s}^{2}\right)^{3 / 2}}\right) .
$$

The right-hand side is proportional to $x_{-}$characteristic for a parametric drive. A simple parametrically driven harmonic oscillator

$$
\ddot{x}+\gamma \dot{x}+\omega_{0}^{2} x=\omega_{0}^{2} K\left[1+2 \epsilon \cos \left(\omega_{D} t\right)\right] x
$$

will be unstable 27 if $\omega_{D}^{-}<\omega_{D}<\omega_{D}^{+}$, where

$$
\omega_{D}^{ \pm}=\omega_{0}\left[2 \sqrt{1-K} \pm \sqrt{\frac{K^{2}}{1-K} \epsilon^{2}-\kappa_{0}^{-2}}\right], \quad \kappa_{0}=\omega_{0} / \gamma,
$$

provided the discriminant is positive, i.e., $\epsilon>\sqrt{1-K} /\left(\kappa_{0} K\right)$. To find the point of instability we keep only the lowest-order term in $x_{-}$in Eq. (14). This gives

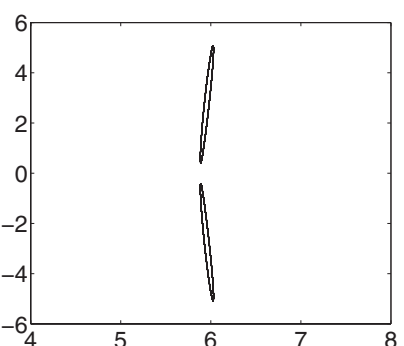

(a)

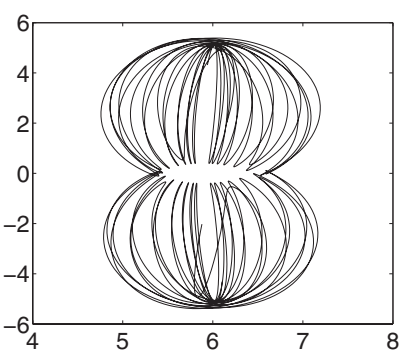

(c)

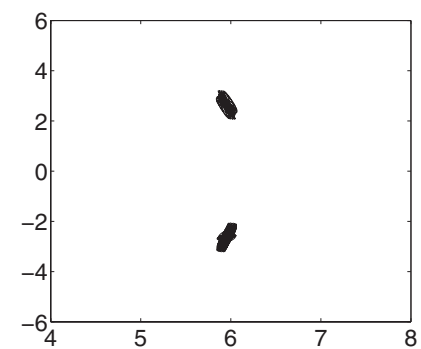

(e)

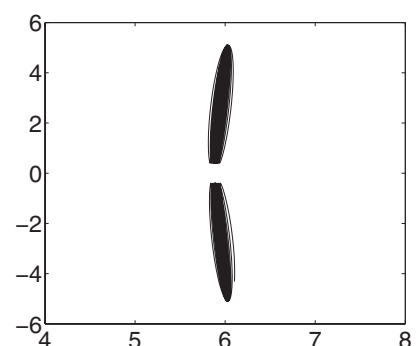

(b)

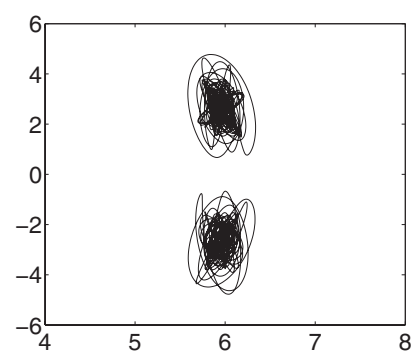

(d)

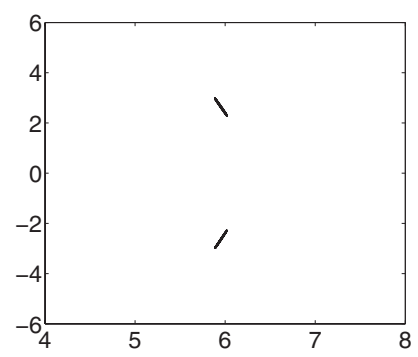

(f)
FIG. 5. Mechanism for destabilization of the high-amplitude branch of the fundamental $y$ resonance (resonance B in Fig. 3). The figure shows the time evolutions of the trajectories of the tubes in the $x y$ plane during destabilization. Initially the tubes are executing high-amplitude motion in the $y$ direction [panel (A)]. The coupling to the motion in the $x$ direction causes instability of the $x_{-}$mode, which starts to grow [panel (B)]. After having reached high amplitude in both $x$ and $y$ directions [panel (C)], irregular motion ensues before the motion finally settles in the low-amplitude branch of the $y$ resonance [panel $(\mathrm{F})]$.

$$
K=2 \nu \frac{\left(2-\Delta_{0}\right)\left(2+\sigma_{s}^{3}\right) x_{s}+\sigma_{s}^{3}}{x_{s}^{2}\left(\Delta_{0}+2+x_{s}^{-1}\right)^{2}},
$$

where $x_{s}$ and $y_{s}$ are the stationary points, $\sigma_{s} \equiv x_{s} / y_{s}$, and $\Delta_{0}=-\Delta_{+}-y^{-1}$. A comparison between numerical simulations and the region of instability is shown in Fig. 7. For small biases the agreement between theory and numerics is good, while it deviates for larger biases. This deviation is due to approximate relations (7) for finding $x_{s}$ and $y_{s}$.

For larger amplitudes we must consider the full equation of motion (14). Introducing action angle coordinates $x_{-}$ $=X(t) \cos [\phi(t)]$ and $\dot{x}_{-}=-X(t) \omega \sin [\phi(t)]$, and expanding $F$ in a Fourier series

$$
F\left(x_{-}\right)=F(X \cos \phi)=a_{0} / 2+\sum_{n=1}^{\infty} a_{n} \cos n \phi,
$$




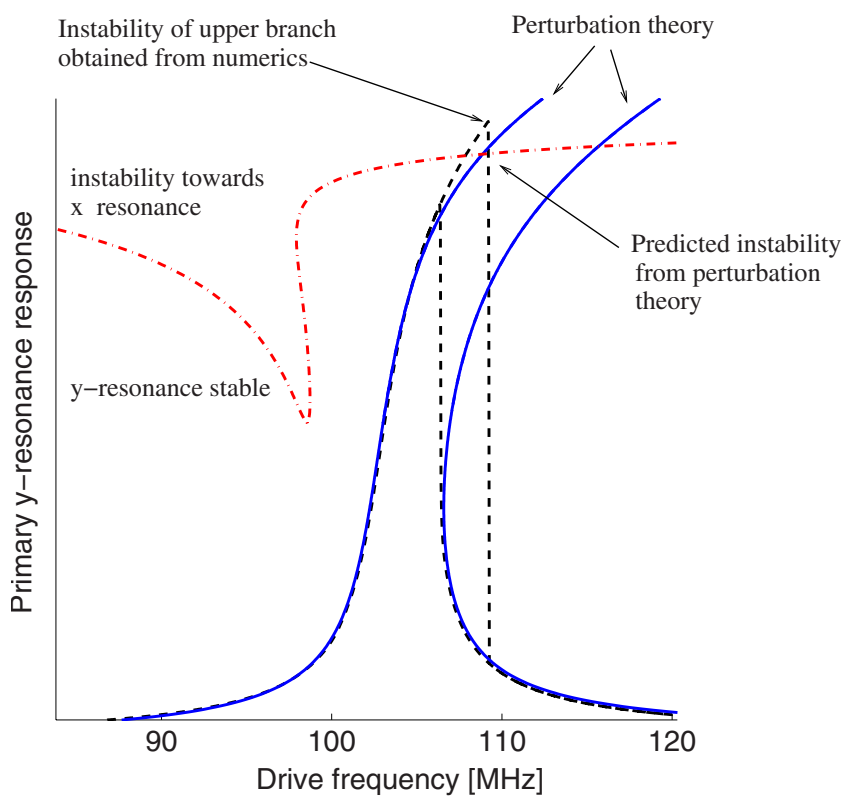

FIG. 6. (Color online) Fundamental $y$ resonance along the dashed line in Fig. 4. The blue solid lines were obtained from solving Eq. (13), while the black dashed lines are from numerical simulations. Above the red dash-dotted line, perturbation theory predicts the fundamental longitudinal resonance to be unstable toward parametric excitation of the $x_{-}$resonance. The point of destabilization of the upper branch occurs where the dash-dotted red line and the upper blue line cross.

we obtain after averaging out fast variables the autonomous system

$$
\begin{aligned}
& \dot{X}=X\left[\epsilon \frac{\omega_{0}^{2}\left(a_{4}-a_{0}\right)}{2 \omega} \sin \delta-\frac{1}{2} \gamma\right], \\
& \dot{\phi}=\omega+\epsilon \frac{\omega_{0}^{2}\left(a_{0}+2 a_{2}+a_{4}\right)}{2 \omega} \cos \delta,
\end{aligned}
$$

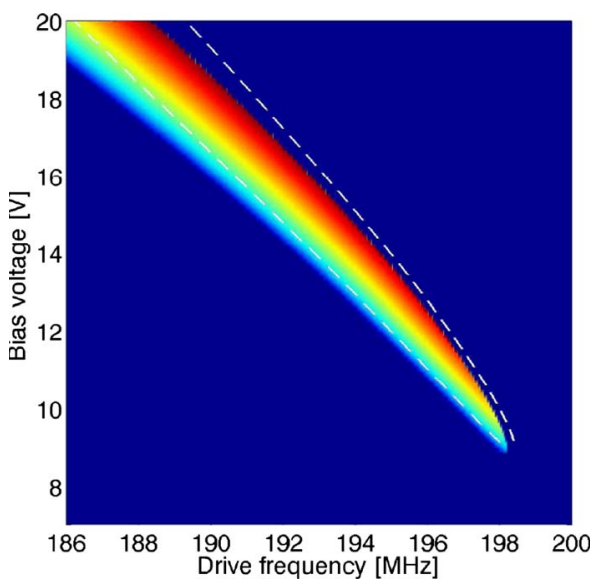

FIG. 7. (Color online) False color plot of the mechanical energy in the parametric $x_{-}$resonance (resonance $\mathrm{C}$ in Fig. 3) as a function of bias voltage (vertical axis) and drive frequency (horizontal axis). The dashed white line denotes the region of instability according to Eqs. (15) and (16).

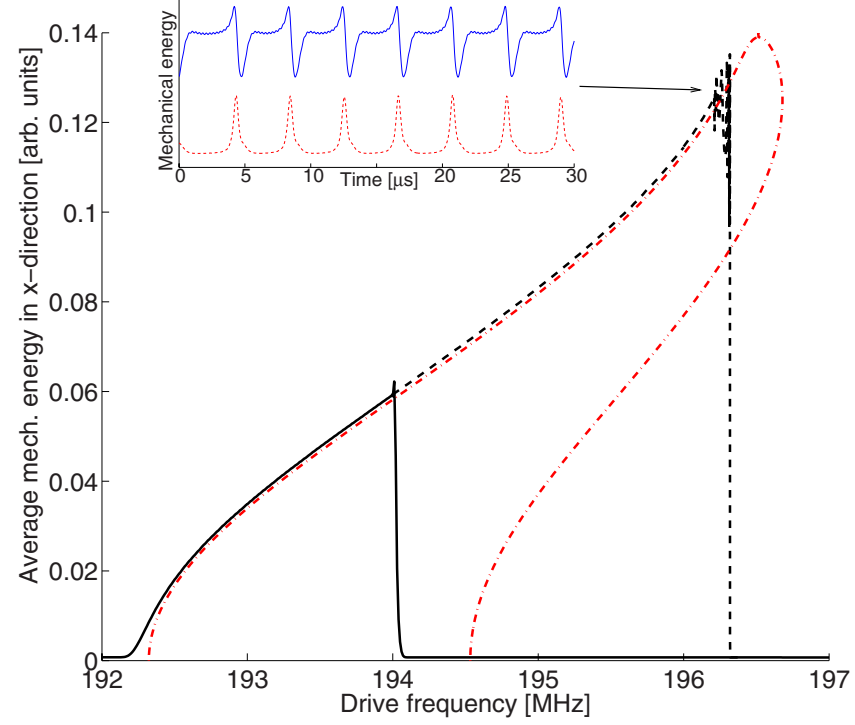

FIG. 8. (Color online) Closeup of the response of the parametric $x_{-}$resonance (cross section at $V_{0}=14 \mathrm{~V}$ in Fig. 7). The resonance has the characteristics of a parametrically driven Duffing resonator with a hardening nonlinearity. Near the point of instability of the upper branch, coupling to the longitudinal mode causes beats where energy is transferred between transverse and longitudinal modes periodically in time. The black curves are from numerical simulations, while the red dash-dotted curves come from solving frequency response equation (17). The inset shows the average mechanical energy stored in transverse and longitudinal modes as function of time. The red curve, showing energy for the longitudinal mode, has been magnified 500 times and vertically displaced for clarity.

$$
\omega=\omega_{0}^{2} \sqrt{1+a_{0}+a_{2}} .
$$

Here $\delta$ is the relative phase of oscillation with respect to the drive. We note that in the limit $X \rightarrow 0$ we have $a_{2}, a_{4}=0$ and $a_{0}=K$. A comparison between the results of perturbation theory and numerical simulation is shown in Fig. 8. Here the mechanical energy in the parametric $x_{-}$resonance is shown for a bias of $V_{0}=14 \mathrm{~V}$ (black solid line is the downward frequency sweep and dashed line is the upward frequency sweep). The red dash-dotted line is the result of solving the frequency response equation

$$
\epsilon^{2} \frac{\omega_{0}^{2}}{\omega^{2}}=\frac{\gamma^{2}}{\left(a_{4}-a_{0}\right)^{2}}+\frac{\left(\omega_{D}-2 \omega\right)^{2}}{\left(a_{0}+2 a_{2}+a_{4}\right)^{2}} .
$$

While agreement between perturbation theory and numerics is good, it does not work well close to the point of instability of the upper branch. Here, there is noise in the curve obtained from numerical simulations. This noise comes from coupling to the longitudinal mode. The inset of Fig. 8 shows how the average energy stored in transverse (blue curve) and longitudinal modes (red curve) vary in time. The curves have been displaced for clarity and the red curve is magnified 500 times. While the energy transferred to the longitudinal mode is very small compared to the energy in the transverse mode, the excited longitudinal vibrations have great impact on the transverse vibrations. 


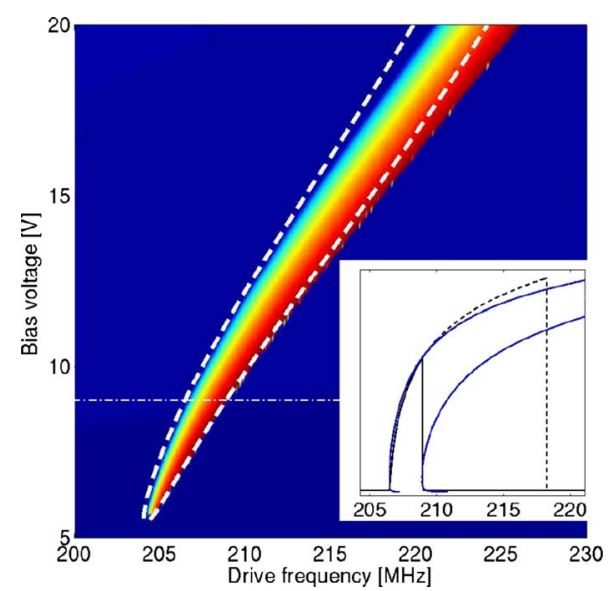

FIG. 9. (Color online) False color plot of the mechanical energy in the parametric longitudinal resonance ( $y$ resonance) as a function of bias voltage (vertical axis) and drive frequency (horizontal axis). The dashed white line denotes the region of instability according to Eqs. (15) and (18). The inset shows the response along the cross section at $9 \mathrm{~V}$ bias (dotted line). The thick black curves come from numerical simulations, and the solid blue lines are solutions to the frequency response Eq. (19).

\section{Parametric longitudinal resonance (y resonance)}

Finally we study the conditions for observing the parametric longitudinal resonance (resonance D in Fig. 3). As in the case of the parametric resonance in the transverse direction, the region of instability in the frequency plane toward parametric resonance in the $y$ direction is determined by Eq. (15). Starting from Eq. (4) and making again the approximations in Eqs. (8) and (9), we find

$$
K \approx-2 \nu A^{2}\left[\alpha^{3}-\frac{2}{\left(y_{s}-A\right)^{3}}\right] \approx \frac{\nu}{y_{s}^{3}}\left[1+\frac{1}{2} \Sigma_{s}\right] .
$$

A comparison between numerical simulations and the region of instability is shown in Fig. 9. The figure was created sweeping the frequency downward and the bifurcation edge is visible as the sharp transition between dark (blue) and bright (red). For small biases the agreement between theory and numerics is good, while it deviates for larger biases. This deviation is again due to using approximate relations (7) to find $x_{s}$ and $y_{s}$.

As in Secs. IV A-IV C we may use perturbation theory to study the large amplitude response of the parametric resonance. Assuming $y(t)=y_{s}+Y \cos \left(\omega_{D} t / 2\right)$ and $v(t)^{2} \approx v_{0}^{2}(1$ $\left.+2 \epsilon \cos \left[\omega_{D} t+\delta\right]\right)$, the frequency response equation can be derived,

$$
\begin{gathered}
\frac{Y^{2}\left(1+\frac{\nu K_{1}}{Y}-\frac{\omega_{D}^{2}}{4 \omega_{0}^{2}}\right)^{2}}{\left(K_{1}+K_{3}\right)^{2}}=\nu^{2} \epsilon^{2}-\frac{\omega_{D}^{2} Y^{2}}{4 \omega_{0}^{2} \kappa_{0}^{-2}\left(K_{3}-K_{1}\right)^{2}}, \\
\tan \delta=\frac{\gamma Y \omega_{D}\left(K_{1}+K_{3}\right)}{\left(K_{3}-K_{1}\right)\left(\left[\omega_{0}^{2}-\omega_{D}^{2} / 4\right] Y-\omega_{0}^{2} \nu K_{1}\right)},
\end{gathered}
$$

where $K_{n}$ are given by Eq. (11). A comparison between perturbation theory and numerical simulations is shown in the

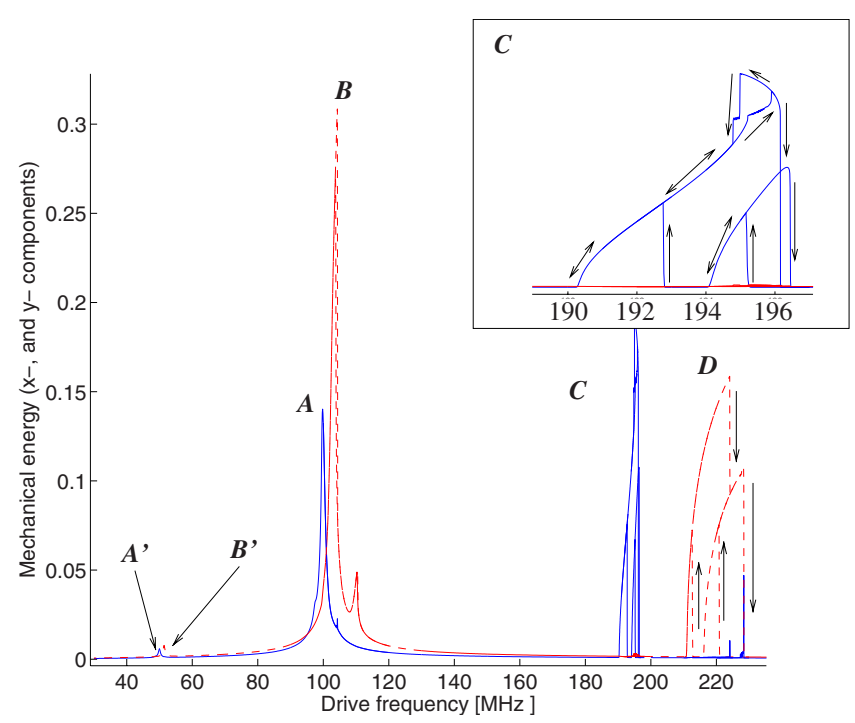

FIG. 10. (Color online) Response of a four-tube system with the same physical parameters as the one in Fig. 3. The inset shows a closeup of the parametric transverse resonance with the directions of the transitions in the frequency plane indicated by arrows.

inset of Fig. 9. Again agreement is good but fails to predict where the upper branch becomes unstable. The destabilization of the parametric $y$ resonance occurs in the same way as the fundamental $y$ resonance, i.e., through parametric excitation of the $x_{-}$mode, and can be analyzed along the lines of reasoning in the Appendix.

\section{SEVERAL OSCILLATORS}

Having treated the two-oscillator system in some detail, we now move on to describe how the system response changes with increasing system size. For this we use the same system parameters (geometry and bias voltages) as those used to obtain Fig. 3 and only change the number of tubes in the array. We have done detailed simulations for systems with 4,8 , and 16 tubes and the corresponding frequency responses are shown in Figs. 10-12.

The fundamental transverse resonance is not markedly affected by the increasing array size. This is expected since here all tubes oscillate in phase with each other. The fundamental longitudinal resonance is however strongly affected, with the single hysteretic peak from the two-tube system developing into a broad band of excited oscillation modes. The presence of this band is reflected also in the longitudinal parametric resonances, where the development of band structure is present in terms of multiple branches and bifurcations in the response. This type of behavior has been seen in parametrically driven NEM and MEM arrays. ${ }^{18}$ Also for the larger arrays large amplitude excitations of longitudinal oscillations can be destabilized due to parametric excitation of transverse modes. In Fig. 12 two particular such points are marked with black circles. At these points the excitation of the transverse modes became so strong that snap-to-contact occurred.

Also the parametric transverse resonance shows the development of a band structure. In contrast to the fundamental 


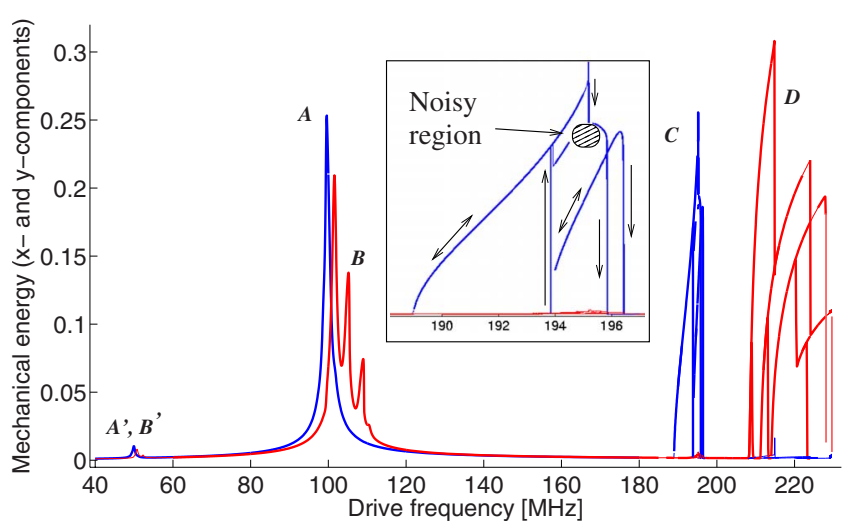

FIG. 11. (Color online) Response of an eight-tube system with the same physical parameters as the one in Fig. 3. The inset shows a closeup of the parametric transverse resonance with the directions of the transitions in the frequency plane indicated by arrows. In the area denoted noisy region, the coupling to longitudinal motion causes the amplitude of transverse motion to oscillate in time.

resonance where this band structure is not accessible, several branches can be reached through parametric excitation. In Figs. 10-12 the insets show closeups of the parametric transverse resonances.

While more and more modes appear as the arrays get larger, one feature is common to all the systems. This feature is the noisy region around $195 \mathrm{MHz}$. In this region, energy is transferred between transverse and longitudinal modes just as in the case of the two-tube system (see Fig. 8) but without destabilizing the transverse motion.

As for the location of the resonances in the voltagefrequency plane, these do not differ appreciably from the two-tube system. The perturbative formulas derived in Sec. IV can be used to estimate if and where the system will be unstable to a certain resonance.

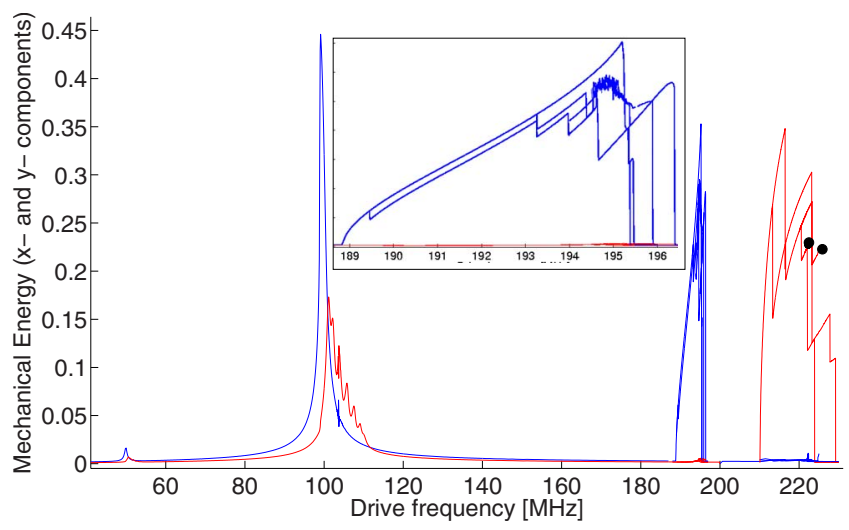

FIG. 12. (Color online) Response of a 16-tube system with the same physical parameters as the one in Fig. 3. The black dots indicate that when the parametric longitudinal resonance became unstable, strong excitations of transverse modes occurred that lead to snap-to-contact of the system. The inset shows a closeup of the parametric transverse resonance. In the area around $195 \mathrm{MHz}$ irregular behavior with high-amplitude motion occurs.

\section{CONCLUSIONS}

In order to investigate the effects of electrostatic interactions between carbon nanotubes in NEM-resonator arrays, we have studied a simple model both analytically and numerically. We have found that, apart from excitation (fundamental and parametric) of a band of longitudinal modes, parametric excitation of transverse modes is also possible. With increasing number of resonators, these resonances become successively more complicated and exhibit rich behavior with several overlapping hysteresis loops, bifurcation points, etc. The transverse modes are also responsible for destabilizing the longitudinal modes at high amplitudes and may lead to snap-to-contact. Also, the parametrically excited transverse modes show regions of irregular behavior coming from coupling between transverse and longitudinal modes. We have shown that the features of the response of 1D arrays can be understood qualitatively through studying the simplest possible array, a two-tube system. Also quantitative predictions based on the two-tube system can be used to obtain estimates of regions of instability toward parametric resonances and to estimate frequency tuning.

From a technological point of view, these estimates can help in designing array resonator systems to avoid unwanted resonances while maintaining a high packing density. Utilizing parametric resonances could also be a path to further increase the operation frequency in technical applications. Moreover by tuning the bias voltages the width of the region of instability can be tuned to an arbitrarily narrow frequency domain. So far, only uniform arrays have been studied. For applications, disorder must be accounted for and further studies are needed.

\section{ACKNOWLEDGMENTS}

This work was supported by the Swedish Foundation for Strategic Research (SSF) and the EU through the Nano-RF Project No. FP6-2005-028158.

\section{APPENDIX: DESTABILIZATION OF PRIMARY $y$ RESONANCE}

We here give a brief derivation of the criteria for destabilization of the fundamental longitudinal mode through parametric excitation of the transverse $x_{-}$mode of the two-tube system. Following the same lines, the stability of the parametric longitudinal excitation can be analyzed.

The longitudinal vibrations are destabilized by the $x_{-}$ mode, which has the equation of motion

$$
\begin{aligned}
\ddot{x}_{-}+\gamma \dot{x}_{-}+\omega_{0}^{2} x_{-}= & \nu \omega_{0}^{2} v^{2}\left[\frac{g_{1}^{2}}{\left(x_{+}+x_{-}\right)^{2}}-\frac{g_{2}^{2}}{\left(x_{+}-x_{-}\right)^{2}}\right. \\
& \left.+2 g_{1} g_{2} \frac{x_{-}}{\left(x_{-}^{2}+y^{2}\right)^{3 / 2}}\right] .
\end{aligned}
$$

For small oscillations of the $x_{-}$mode, the right-hand side can be approximated for large amplitudes $Y$ of the $y$ mode (recalling that $\left.y=y_{s}+Y \cos \omega_{D} t\right)$, yielding the equation 


$$
\ddot{x}_{-}+\gamma \dot{x}_{-}+\omega_{0}^{2} x_{-}=-x_{-} F(t),
$$

where

$$
F(t) \equiv \frac{2 \nu A^{2} \omega_{0}^{2} v_{0}^{2}\left[1+2 \epsilon \cos \left(\omega_{D} t+\delta\right)\right]}{\left[y_{s}+Y \cos \left(\omega_{D} t\right)\right]\left[Y \cos \left(\omega_{D} t\right)+y_{s}-A\right]^{2}},
$$

and we have defined $A \equiv\left(2+1 / x_{s}-\alpha\right)^{-1}$ and $\alpha \equiv\left(x_{s}^{2}\right.$ $\left.+y_{s}^{2}\right)^{-1 / 2}$. Changing to action angle variables $\left(x_{-}\right.$ $=X(t) \cos [\phi(t)]$ and $\left.\dot{x}_{-}=-X(t) \omega \sin [\phi(t)]\right)$ and averaging over fast variables results in the autonomous system

$$
\begin{gathered}
\dot{X}=X\left[\frac{\langle\sin 2 \phi F(t)\rangle}{2 \sqrt{\omega_{0}^{2}+c_{0}}}-\frac{\gamma}{2}\right], \\
\dot{\phi}=\sqrt{\omega_{0}^{2}+c_{0}}+\frac{1}{2 \sqrt{\omega_{0}^{2}+c_{0}}}\langle\cos 2 \phi F(t)\rangle,
\end{gathered}
$$

where the brackets denotes the averaging $\langle\cdot\rangle \equiv(2 \pi)^{-1} \int_{0}^{2 \pi} \cdot d \phi$, and we have expanded $F(t)$ in a Fourier series $F(t)$ $=\Sigma_{n} c_{n} e^{i n \omega_{D} t}$. At the onset of the destabilizing $x_{-}$resonance we have $\dot{\phi}=\omega_{D}$ and $\phi=\omega_{D} t+\vartheta$. Evaluating the averages and setting $c_{2}=\left|c_{2}\right| e^{i \lambda}$, one finds

$$
\begin{gathered}
\dot{X}=X\left[\left|c_{2}\right| \frac{\sin (2 \vartheta-\lambda)}{2 \sqrt{\omega_{0}^{2}+c_{0}}}-\frac{\gamma}{2}\right], \\
\omega_{D}-\sqrt{\omega_{0}^{2}+c_{0}}=\frac{1}{2 \sqrt{\omega_{0}^{2}+c_{0}}}\left|c_{2}\right| \cos (2 \vartheta-\lambda) .
\end{gathered}
$$

The region of driving frequencies where the high-amplitude branch of the $y$ mode can be destabilized by the $x_{-}$mode can then be found as $\omega_{D}^{\text {destab- }}<\omega_{D}<\omega_{D}^{\text {destab+ }}$, with

$$
\omega_{D}^{\mathrm{destab} \pm}=\left[\sqrt{\omega_{0}^{2}+c_{0}} \pm \sqrt{\frac{\left|c_{2}\right|^{2}}{4\left(\omega_{0}^{2}+c_{0}\right)}-\frac{\gamma^{2}}{4}}\right] .
$$

Using expression (A1) the Fourier coefficients $c_{0}$ and $c_{2}$ can be evaluated exactly:

$$
\begin{gathered}
\frac{c_{0}}{\omega_{0}^{2}}=2 \nu\left[\frac{Y+2\left(A-y_{s}\right) \epsilon \cos \delta}{Y \sqrt{-Y^{2}+\left(A-y_{s}\right)^{2}}}-\frac{Y+2 \epsilon y_{s} \cos \delta}{Y \sqrt{-Y^{2}+y_{s}^{2}}}\right. \\
\left.+\frac{A\left(A-y_{s}\right)+2 A Y \epsilon \cos \delta}{\left[-Y^{2}+\left(A-y_{s}\right)^{2}\right]^{3 / 2}}\right], \\
\frac{c_{2}}{\omega_{0}^{2}=} \nu\left[\frac{\left[Y^{2}+\left(A-y_{s}\right) y_{s}+A(2 Y) \epsilon \cos \delta\right]}{\left[-Y^{2}+\left(A-y_{s}\right)^{2}\right]^{3 / 2}}\right. \\
\left.-2 \frac{Y\left(A-y_{s}\right) y_{s}-\left[y_{s} Y^{2}-2\left(A+y_{s}\right)\left(A-y_{s}\right)^{2}\right] \epsilon \cos \delta}{Y^{3} \sqrt{-Y^{2}+\left(A-y_{s}\right)^{2}}}\right] \\
\left.+\frac{\left(Y^{2}-2 y_{s}^{2}\right)\left(Y-2 y_{s} \epsilon \cos \delta\right)}{Y^{3} \sqrt{-Y^{2}+y_{s}^{2}}}+\frac{4 A^{2} \epsilon \cos \delta}{Y^{3}}\right] .
\end{gathered}
$$

After solving the frequency response equation for the $y$ resonance, $Y$ and $\cos \delta$ can be found and the expressions can be evaluated, thus determining whether or not parametric excitation of the $x_{-}$mode will occur. *andreas.isacsson@chalmers.se

${ }^{1}$ V. Sazonova, Y. Yaish, H. Üstünel, D. Roundy, T. A. Arias, and P. L. McEuen, Nature (London) 431, 284 (2004).

${ }^{2}$ H. B. Peng, C. W. Chang, S. Aloni, T. D. Yuzvinsky, and A. Zettl, Phys. Rev. Lett. 97, 087203 (2006).

${ }^{3}$ S. T. Purcell, P. Vincent, C. Journet, and V. T. Binh, Phys. Rev. Lett. 89, 276103 (2002).

${ }^{4}$ B. Witkamp, M. Poot, and H. S. J. van der Zant, Nano Lett. 6, 2904 (2006).

${ }^{5}$ D. Garcia-Sanchez, A. San Paulo, M. J. Esplandiu, F. PerezMurano, L. Forro, A. Aguasca, and A. Bachtold, Phys. Rev. Lett. 99, 085501 (2007).

${ }^{6}$ A. Eriksson, S.-W. Lee, A. A. Sourab, A. Isacsson, R. Kaunisto, J. M. Kinaret, and E. E. B. Campbell, Nano Lett. 8, 1224 (2008).

${ }^{7}$ C. Hierold, J. Micromech. Microeng. 14, S1 (2004).

${ }^{8}$ M. Roukes, Phys. World 14 (2), 25 (2001).

${ }^{9}$ A. N. Cleland, Foundations of Nanomechanics (Spinger-Verlag, Berlin, 2003).

${ }^{10}$ International Technology Roadmap for Semiconductors (ITRS), 2007 edition (http://www.itrs.net/reports.html).

${ }^{11}$ M. Sato, B. E. Hubbard, A. J. Sievers, B. Ilic, D. A. Czaplewski, and H. G. Craighead, Phys. Rev. Lett. 90, 044102 (2003).
${ }^{12}$ M. Sato, B. E. Hubbard, L. Q. English, A. J. Ilic, D. A. Czaplewski, and H. G. Craighead, Chaos 13, 702 (2003).

${ }^{13}$ M. Sato, B. E. Hubbard, and A. J. Sievers, Rev. Mod. Phys. 78, 137 (2006).

${ }^{14}$ W. J. Venstra and H. S. J. van der Zant, Appl. Phys. Lett. 93, 234106 (2008).

${ }^{15}$ M. C. Cross, A. Zumdieck, R. Lifshitz, and J. L. Rogers, Phys. Rev. Lett. 93, 224101 (2004).

${ }^{16}$ M. C. Cross, J. L. Rogers, R. Lifshitz, and A. Zumdieck, Phys. Rev. E 73, 036205 (2006).

${ }^{17}$ E. Buks and M. L. Roukes, Europhys. Lett. 54, 220 (2001).

${ }^{18}$ E. Buks and M. L. Roukes, J. Microelectromech. Syst. 11, 802 (2002).

${ }^{19}$ R. Lifshitz and M. C. Cross, Phys. Rev. B 67, 134302 (2003).

${ }^{20}$ Y. Bromberg, M. C. Cross, and R. Lifshitz, Phys. Rev. E 73, 016214 (2006)

${ }^{21}$ J. Zhu, C. Q. Ru, and A. Mioduchowski, Eur. Phys. J. B 58, 411 (2007).

${ }^{22}$ J. A. Pelesko and D. H. Bernstein, Modeling MEMS and NEMS (Chapman and Hall, London/CRC, Boca Raton, FL, 2003).

${ }^{23}$ A. Isacsson, R. Kaunisto, and J. M. Kinaret, Nanotechnology 18, 195203 (2007)

${ }^{24}$ H. Jiang, M.-F. Yu, B. Liu, and Y. Huang, Phys. Rev. Lett. 93, 
185501 (2004).

${ }^{25} \mathrm{~K}$. Engström (unpublished).

${ }^{26}$ B. Peng, M. Locascio, P. Zapol, S. Li, S. L. Mielke, G. C. Schatz, and H. D. Espinosa, Nat. Nanotechnol. 3, 626 (2008).
${ }^{27}$ A. H. Nayfeh and D. T. Mook, Nonlinear Oscillations (WileyVCH, Birkach, Germany, 2004).

${ }^{28}$ A. H. Nayfeh, Introduction to Perturbation Techniques (Wiley Interscience, New York, 1993). 